\title{
Linfoma de Hodgkin con compromiso hepático tratado con esquema gemcitabina, dexametasona y cisplatino como puente para terapia estándar. Caso clínico
}

'Servicio de Hematología, Hospital Regional de Talca, Talca, Chile.

aAlumno(a) Facultad de Medicina Universidad Católica del Maule, Talca, Chile.

Recibido el 31 de agosto de 2011, aceptado el 17 de enero de 2012.

Correspondencia a: Matías Orellana Escuela de Medicina, Facultad de Medicina. Universidad Católica del Maule. Av. San Miguel № 3605, Talca, Chile.

Fax: 56-71-413657 E-mail:m.orellana.ucm@ gmail.com

\section{Hodgkin lymphoma with hepatic involvement treated with dexametasone, gemcitabine and cisplatin as a bridge to standard therapy. Report of one case}

The initial presentation of Hodgkin lymphoma with liver involvement is rare. In these patients, the standard first-line therapy with ABVD (Adriamycin, Bleomycine, Vinblastine, Dacarbazine) imply an additional risk for liver toxicity. We report a 64-year-old woman who presented with jaundice, choluria, malaise and weight loss. In the initial evaluation she had jaundice and palpable groin lymph nodes. An obstructive biliary disease was ruled out with magnetic resonance imaging studies. A lymph node biopsy showed a Hodgkin's lymphoma, Mixed-cellularity subtype. Considering the liver dysfunction, an alternative scheme of chemotherapy with dexamethasone, gemcitabine and cisplatin (GDP) was administered. After 4 cycles, a significant improvement in liver hepatic function tests was reached and a conventional chemotherapy ( $A B V D)$ was begun. While the literature provides some low toxicity protocols for patients with liver involvement, favorable results of our clinical case report allows us to postulate GDP as an alternative for salvage therapy in these patients.

(Rev Med Chile 2012; 140: 902-905).

Key words: Antineoplastic Combined Chemotherapy Protocols; Cisplatin; Drug induced liver injury; Hodgkin disease.
$\mathrm{E}$ 1 linfoma de Hodgkin (LH) se presenta generalmente como adenopatías no dolorosas ${ }^{1,2}$; $\checkmark$ menos común es la presentación inicial con compromiso hepático colestásico ${ }^{1,3,4}$, ocurriendo esta normalmente en etapas tardías o en el curso final de la enfermedad ${ }^{2}$. En el laboratorio se manifiesta con elevación de las transaminasas y la bilirrubina $^{3}$. En raras ocasiones se ha atribuido al LH la causa de una falla hepática fulminante, de muy mal pronóstico ${ }^{1}$.
El compromiso hepático se produce por 4 mecanismos: i) la infiltración del parénquima por el tumor; ii) la obstrucción extrínseca de la vía biliar extrahepática; iii) menos frecuentemente se produce una ductopenia o síndrome del ducto hepático evanescente supuestamente causado por citoquinas y por último; iv) la presentación paraneoplásica idiopática, no asociada a los causas anteriores ${ }^{1}$.

La terapia con esquema ABVD (doxorrubicina, 
bleomicina, vinblastina y dacarbazina) podría agregar un factor adicional de toxicidad hepática en este caso, lo que plantea un dilema en el tratamiento inicial de estos pacientes ${ }^{5}$.

A continuación se presenta el caso de una paciente portadora de un linfoma Hodgkin con compromiso hepático, que es tratada con el esquema GDP (gemcitabina, dexametasona, cisplatino), el cual posee menos hepatotoxicidad ${ }^{1,5-7}$, se puede utilizar a dosis plenas ${ }^{1} \mathrm{y}$ ha mostrado efectividad en esta patología ${ }^{7-9}$.

\section{Caso clínico}

Mujer de 64 años con antecedentes de epilepsia, neurocisticercosis, accidente vascular isquémico, úlcera péptica y colecistectomía 5 años previo al ingreso.

Consultó por ictericia, coluria, acolia, anorexia y baja de peso importante durante el último mes, sin otras molestias. En el examen físico se evidenció una marcada ictericia de piel con adenopatías inguinales palpables.

En el laboratorio se encontró anemia (hemoglobina 7,2 g/dl), elevación de transaminasas (GOT $286 \mathrm{U} / \mathrm{L}, \mathrm{GPT} 177 \mathrm{U} / \mathrm{L}$ ) y fosfatasas alcalinas (1.420 $\mathrm{U} / \mathrm{L})$, hiperbilirrubinemia de predominio directo (total $18,84 \mathrm{mg} / \mathrm{dl}$, directa $18,75 \mathrm{mg} / \mathrm{dl}$ ), hipoalbuminemia (2,3 g/dl), hipercolesterolemia (524 mg/ dl), concentración de protrombina $8,9 \%$, tiempo de protrombina de 84,9 segundos, recuento plaquetario de $415^{\star} 10^{\wedge} 3 /$ uL y función renal normal.

Se realizó estudio de vía biliar con ecografía, colangiografía retrógrada endoscópica y resonancia nuclear magnética que descartaron posible obstrucción intrínseca o extrínseca.

La tomografía axial computarizada de abdomen mostró hepatomegalia y múltiples adenopatías intraabdominales e inguinales de aspecto inespecífico.

La biopsia de ganglio inguinal y de médula ósea concluyó linfoma de Hodgkin tipo clásico, variedad celularidad mixta, con positividad para CD30 y CD15, y ausencia de CD20 en las células neoplásicas.

Considerando su insuficiencia hepática y la hepatotoxicidad de los esquemas convencionales, se inició tratamiento con corticoides y luego con CHOP (ciclofosfamida, adriamicina, vincristina, prednisona), con regular respuesta clínica, per- sistiendo la ictericia y los signos de disfunción hepática (GOT 263 U/L, GPT 256 U/L, fosfatasas alcalinas $2.979 \mathrm{U} / \mathrm{L}$, bilirrubina total $8 \mathrm{mg} / \mathrm{dl}$ ), por lo que se cambió quimioterapia a esquema GDP realizándose 4 ciclos. Como complicación presentó 2 episodios de sepsis por E. coli tratados en forma exitosa. Se logró finalmente una disminución efectiva de los niveles de bilirrubina (total $1,73 \mathrm{mg} / \mathrm{dl}$, directa $1,26 \mathrm{mg} / \mathrm{dl}$ ), lo que permitió el uso de la terapia estándar (ABVD).

Actualmente, 75 días tras fin del último ciclo de GDP, se encuentra anictérica, cursando el segundo ciclo de ABVD, mostrando una buena tolerancia. El laboratorio muestra anemia moderada (hemoglobina $9,1 \mathrm{~g} / \mathrm{dl}$ ), menor elevación de transaminasas (GOT (90 U/L), GPT $55 \mathrm{U} / \mathrm{L}$ ) y fosfatasas alcalinas (698 U/L), con normalización de la bilirrubina (total $1,34 \mathrm{mg} / \mathrm{dl}$, directa $0,7 \mathrm{mg} /$ dl) y función renal normal.

\section{Discusión}

Nuestra comunicación presenta una nueva alternativa de tratamiento para un grupo de pacientes que, si bien es reducido, plantea al médico un gran desafío.

Es sabido que muchos fármacos pueden producir daño hepático y que la mayoría de estas reacciones son idiosincráticas ${ }^{6}$. Sin embargo, los efectos hepatotóxicos de la quimioterapia en general son escasos, siendo provocados sólo por algunos agentes ${ }^{6}$.

El esquema ABVD es el elegido como terapia inicial en el tratamiento del $\mathrm{LH}^{7,10-12}$, con buenos resultados ${ }^{10,12,13}$. Sin embargo, el riesgo de hepatotoxicidad existe, habiéndose reportado desde leve alza de transaminasas hasta un caso de síndrome de Budd-Chiari, que resultó mortal ${ }^{13}$.

La doxorrubicina, antibiótico de la familia de las antraciclinas, es metabolizada en el hígado. Se ha descrito que este fármaco, en combinación con otros y posiblemente sólo, puede causar necrosis hepática ${ }^{13}$. En una serie de 6 pacientes con diagnóstico de leucemia linfoblástica aguda en terapia con doxorrubicina, vincristina y prednisona, se presentaron elevaciones de transaminasas hepáticas GOT y GPT al poco tiempo del inicio del tratamiento ${ }^{14}$. Se recomienda un ajuste de dosis en estos casos aun cuando la hepatotoxicidad fatal por doxorrubicina es rara $^{6,13,15}$. 
La bleomicina es inactivada por una aminopeptidasa presente en el hígado y otros tejidos, y excretada por la orina. Todavía no hay consenso si produce daño hepático importante ${ }^{6}$. En una revisión del año 1973 donde fueron tratados con bleomicina más de 1.000 pacientes, no hubo reportes de daño hepático severo ${ }^{16}$. Pese a esto, debido a su metabolismo hepático, debe considerarse la función hepática para evaluar la necesidad de ajustar la dosis.

La dacarbazina es metabolizada por vía microsomal hepática y ha tenido reportes de toxicidad hepática vascular en pacientes tratados sólo con esta droga ${ }^{6}$.

Considerando lo anteriormente expuesto, se hace necesario el uso de esquemas alternativos, que generalmente incluyen al cisplatino combinado con otros agentes 5 . Si bien en la literatura se encuentran algunos reportes de caso que sugieren el esquema DHAP (cisplatino, citarabina y dexametasona) como alternativa inicial y sin ajuste de dosis en pacientes con LH y disfunción hepática ${ }^{5}$, en nuestra paciente nos planteamos el esquema GDP como terapia de rescate, principalmente debido a su efectividad en pacientes en recaída y baja hepatotoxicidad potencial.

El esquema GDP ha sido usado en LH refractarios a tratamiento ${ }^{8,9}$. En una serie de 17 pacientes se encontró una respuesta global de $94 \%$ y completa de $65 \%$, principalmente en el grupo que recibió 3 ciclos $^{12}$. En otra serie de 17 pacientes se encontró una respuesta global de $70 \%$, incluyendo 4 respuestas completas, obteniéndose además dosis adecuadas de células madre para el trasplante autólogo. Las principales toxicidades grado 3 y 4 descritas fueron de tipo hematológico, especialmente neutropenia $(8,6 \%)$ y trombocitopenia $(13 \%)^{17}$. El cisplatino rara vez causa hepatotoxicidad (con esteatosis y colestasis) a dosis convencionales, sin embargo, sí podría ocurrir a dosis elevadas 7 . La gemcitabina, un análogo de las pirimidinas, puede causar aumento de transaminasas, que es transitorio y sin repercusiones clínicas ${ }^{6}$. En estudios fase II se ha usado como monoterapia en LH, lográndose respuestas que van de $39^{18}$ al $43 \%{ }^{19}$ pero de corta duración, por lo que se ha buscado optimizar los resultados asociándola con otros agentes ${ }^{7}$.

A pesar de lo infrecuente que el LH se presente con compromiso hepático importante, nuestro reporte muestra la necesidad de contar con esquemas de quimioterapia alternativos y de baja toxicidad para estos pacientes. En la literatura revisada, encontramos reportes de uso de $\mathrm{CHOP}$, MOPP (mecloretamina, vincristina, procarbazina, prednisona) y DHAP con resultados positivos ${ }^{4,5,13}$. En la paciente del caso planteado se eligió como primera aproximación terapéutica el uso de CHOP, el que debió suspenderse por el deterioro clínico y de laboratorio tras su inicio. Esto contrasta con el caso reportado de una paciente que se presenta con compromiso hepático que fue tratada inicialmente con CHOP de manera exitosa ${ }^{4}$. Aunque, es importante destacar que esta paciente presentaba menor compromiso sistémico y no poseía comorbilidades, lo que nos lleva a plantear la importancia de la elección y adecuación del esquema según cada paciente.

Es importante señalar que el estudio de nuestra paciente no cuenta con confirmación histológica por biopsia hepática. Los motivos para la no realización de esta fueron principalmente el gran compromiso clínico que presentaba la paciente para ser sometida a un procedimiento invasivo, que en el estudio diagnóstico no se encontró evidencia de alguna otra patología actual que explicara el compromiso hepático, y la buena respuesta clínica y de laboratorio al inicio de la terapia con GDP. Por esto, si bien el estudio no se encuentra completo, la realización de la biopsia hepática no hubiera alterado la conducta clínica.

En conclusión, el caso que presentamos plantea la terapia con GDP como una alternativa de manejo posible y efectiva en pacientes con LH que se presentan con gran compromiso hepático. Este esquema, de acuerdo a nuestro conocimiento, no ha sido descrito a la fecha como terapia inicial en el contexto de la insuficiencia hepática por linfoma de Hodgkin.

\section{Referencias}

1. Hong F, Smith C, Angus P, Crowley P, Khoon Ho W. Hodgkin lymphoma and fulminant hepatic failure. Leukemia \& Lymphoma 2010; 51 (5): 947-51.

2. Chim C, Choy C, Ooi C, Liang R. Hodgkin's Disease with Primary Manifestation in the Liver. Leukemia \& Lymphoma 2000; 37 (5-6): 629-32.

3. Shehab T, Kaminski M, Lok A. Acute Liver Failure Due to Hepatic Involvement by Hematologic Malignancy. Digestive Diseases and Sciences 2008; 42 (7): 1400-5.

4. Serrano-Navarro I, Rodríguez-López J, Navas-Espejo 
R, Pérez-Jacoiste M, Martínez-Gonzáloz M, Grande C, et al. Linfoma hepático primario. Evolución favorable con quimioterapia combinada con Rituximab. Revista Española de Enfermedades Digestivas 2008; 100 (11): 724-8.

5. McCarthy j, Gopal A. Successful Use of Full-Dose Dexamethasone, High-Dose Cytarabine, and Cisplatin as Part of Initial Therapy in Non-Hodgkin Lymphoma with Severe Hepatic Dysfunction. Clinical Lymphoma \& Myeloma 2009; 9 (2): 167-70.

6. King P, Perry M. Hepatotoxicity of Chemotherapy. The Oncologist 2001; 6: 162-76.

7. Oki Y, Younes A. Current role of gemcitabine in the treatment of Hodgkin lymphoma. Leukemia \& Lymphoma 2008; 49 (5): 883-9.

8. Todd T, Raj D, Camilleri D, Stafford G, Bulusu R, Follows $\mathrm{G}$, et al. (2009). Intermediate dose gemcitabine-cisplatin combination chemotherapy without treatment delay for cytopenia followed by autografting-a new standard of care in relapsed or refractory Hodgkin lymphoma? Ann Hematol 2008; 88: 1107-12.

9. Kuruvilla J, Nagy T, Pintilie M, Tsang R, Keating A, Crump M. Similar Response Rates and Superior Early Progression-Free Survival with Gemcitabine, Dexamethasone, and Cisplatine Salvage Therapy Compared with Carmustine, Etoposide, Cytarabine, and Melphalan Salvage Therapy Prior to Autologous Stem Cell Transplation for Recurrent or Refractory Hodgkin Lymphoma. Cancer 2006; 106 (2): 353-60.

10. Glossmann J, Josting A, Diehl V. New Treatments for
Hodgkin's Disease. Lymphomas 2002; 3: 283-90.

11. Bartlett N. Modern treatment of Hodgkin lymphoma. Lymphoid biology and diseases 2008; 15: 408-14.

12. Evens M, Hutchings M, Diehl V. Treatment of Hodgkin Lymphoma: the past, present, and future. Oncology 2008; 5(9): 543-556.

13. Joensuu H, Söderstrôm K, Nikkanen V. Fatal Necrosis of the Liver During ABVD Chemotherapy for Hodgkin's Disease. Cancer 1986; 58: 1437-40.

14. Aviles A, Herrera J, Ramos E, Ambriz J, Pizzuto J. Hepatic Injury during doxorrubicin therapy. Arch Pathol Lab Med 1984;108: 912-3.

15. Aapro M, Martin C, Hatty S. Gemcitabine: a safety review. Anticancer drugs 1998; 9: 191-201.

16. Blum R, Carter S, Agre K. A clinical review of bleomycin. A new antineoplasic agent. Cancer 1973; 31: 903-14.

17. Santoro A, Bredenfeld H, Devizzi L, Tesch H, Bonfante $\mathrm{V}$, Viviani F, et al. Gemcitabine in the treatment of refractory Hodgkin's disease: result of multicenter phase II study. J Clin Oncol 2000;18: 2515-619.

18. Zinzani PL, Bendandi M, Stefoni V, Albertini P, Gherlinzoni F, Tani M, et al. Value of gemcitabine treatment in heavily pretreated Hodgkin's disease patients. Haematologica 2000; 85 (9): 926-9.

19. Baetz T, Belch A, Couban S, Imrie K, Yau J, Myers R, et al. Gemcitabine, dexamethasone and cisplatin is an active and non-toxic chemotherapy regimen in relapsed or refractory Hodgkin's disease: a phase II study by the National Cancer Institute of Canada Clinical Trials Group. Ann Oncol 2003; 14 (12): 1762-7. 